ORIGINAL ARTICLE

\title{
Recipients Perception of Blood Transfusion Risk in Some Selected Tertiary Care Hospitals
}

\author{
*MM Rahman ${ }^{1}$, TT Sajani ${ }^{2}$ \\ ${ }^{1}$ Prof. Dr. Md. Mahfuzar Rahman, Head, Department of Community Medicine, AKMMC. \\ ${ }^{2}$ Dr. Tabassum Tahmin Sajani, Assistant Professor, Department of Community Medicine, AKMMC \\ *Corresponding Author
}

\begin{abstract}
This was a descriptive study carried out in the Medicine, Surgery, Gynae and Orthopedics ward of three randomly selected tertiary care hospitals at Dhaka, Bogra and Rangpur. It was aimed to evaluate the extent of understanding about the risk of blood transfusion among the blood recipients, to identify the common indications for blood transfusion through record review, to determine the recipients knowledge about indications of blood transfusion, their level of understanding about blood group, blood donors, and risk of blood transfusion and to find out the socio-demographic condition of recipients admitted at tertiary care hospitals. It was found that most of the respondents $63.17 \%$ were in the age group of $15-39$ years and $56.95 \%$ were between primary to secondary level of education. More than half $56.83 \%$ respondents were housewife. The idea about indications of blood transfusion was found among $60.37 \%$ respondents. The idea about own blood group was found among $73.54 \%$ respondents. However, $52.93 \%$ respondents were unaware about the persons who can donate blood. Professional blood donor were the choice for own to only $15.60 \%$ respondents. The study also identified $57.32 \%$ respondents having risk of blood transmissible diseases. The respondents idea about transmissible diseases were identified as AIDs, Hepatitis-B, Syphilis and Malaria by $90.48 \%, 42.38 \%, 5.25 \%$ and $5.25 \%$ respectively. The study demands awareness and motivational program among younger and adult females in regards to transfusion transmissible diseases and its prevention. The importance of knowing blood group,donor selection and adverse effects of transfusion may also considered as a part of awareness program. It also demands a large scale community based survey to generate information towards development of effective strategy in minimizing risk of transfusion transmissible diseases in particular.
\end{abstract}

Key words: Blood recipients, Perception, Blood transfusion

\section{Introduction}

Blood transfusion is an essential service for modern medical treatment. There is no substitute for blood. Its requirement in so many emergencies like accidents, acute surgery, acute medical emergency, burn and also in hematological conditions like thalassaemia, leukaemia, aplastic anaemia etc is essential. So it has got a great impact in health care delivery system. Transfusion of one unit blood may add att an average of 350-400 $\mathrm{ml}$ of blood. The transfusion of one unit of red cells generally raises the hemoglobin by $10 \mathrm{~g} / 1 .{ }^{1}$
The WHO has set the goal that every country in the world should have supplies of blood and blood products that are safe as possible, accessible to reasonable cost and adequate to meet national need. In Bangladesh about 2-2.5 lacs units of blood is required yearly. Paid blood donors share the major portion $(70 \%)$ of collected blood in the country suffering from Hepatitis B(29\%), Hepatitis C $6 \%)$ and Syphilis (22\%) significantly. ${ }^{2}$

Blood transfusion can be life saving but in some instances it can cause dangerous harm to the 
blood recipients. So it should not be given without definite indications. Risks and adverse events among recipients of blood transfusion includes both transfusion of infectious diseases and noninfectious adverse events. The later may occur as a result of errors in handling or administering blood and blood products. Currently, as a result of donor screening and new test procedures, the risk of transmission of known infectious agents is extremely low, however because blood is a biological product, unknown and emerging pathogens will always pose threat to its safety.

Major portion of the total blood is collected in Bangladesh by different hospitals, institutes, Non government teaching hospitals, Redcrescent, Sandhani, Lions, Rotarian and private clinics. A small portion of total blood is collected by commercial blood banks. There is lack of coordination in terms of safe collection, proper storage, distribution and screening of blood among Govt. Hospital and other organizations. As a result presents system of blood donation causes wastage of significant portion of collected blood and increase risk of transfusion transmissible diseases (TTD).

The study is a modest attempt to explore the understanding of blood recipients about blood group, donor's characteristics and the risks associated with blood transfusion. The findings of the study may help formulating an intervention strategy to avoid unwanted risks associated with blood transfusion and improve awareness program for the recipients's taking the blood transfusion in addition to the creation of donor's awareness. Ultimately this will help in minimizing transfusion transmissible disease like hepatitis $\mathrm{B}$ and HIV/AIDS in the country in particular.

\section{Methodology}

A cross sectional study was done in Medicine, Surgery, Gynae and Orthopedics ward of three randomly selected tertiary care hospitals at Dhaka, Bogra and Rangpur from the period of September 2007 to February 2008. The entire admitted recipient's of blood transfusion during data collection period were considered in the sampling frame and included in the study as key informants. Data were collected by self administered questionnaire and recipients record review during hospital office hours. The indication of blood transfusion and sociodemographic information were collected from blood recipient's admission record. After collection, data were edited for any inconsistency, coded, compiled and tabulated. It was processed by scientific calculator and computer using appropriate soft ware program. The analysis was made with appropriate statistical interpretation towards report writing.

\section{Results}

A total of 820 respondents in three tertiary care hospital (Dhaka, Bogra and Rangpur) were interviewed. Most of the respondents $67.93 \%$ were female of which $63.17 \%$ were in the age group $15-39$ years. $56.95 \%$ respondent's level of education was between primary and secondary level. More than half of the respondents $56.83 \%$ were housewife and majority $86.59 \%$ of them were married (Table1) and $31 \%$ respondents monthly income was within 2001 to 3000 taka. (Figure-1)

Idea about indication of blood transfusion were revealed among $60.37 \%$ respondents. However, the nature of idea on indications of blood transfusion were identified as severe bleeding, anaemia and operation to $14.34 \%, 22.63 \%$ and $29.29 \%$ respondents respectively. (Table-2)

Only $33.41 \%$ respondents identified the nature of usual examination performed before transfusion as blood grouping. Idea about own blood group was found to $73.54 \%$ respondents. However, $52.93 \%$ respondents were not aware about the persons who can donate blood. (Table-2) Around $52.93 \%$ respondents had no idea about frequency of blood donation in a year. (Figure-2) Professional blood donors were the choice for own to $15.60 \%$ respondents only. However reasons for discouraging professional blood donors were identified as risk of blood transmissible disease by $57.32 \%$ respondents respectively. (Table-3) Around $74.39 \%$ respondents were having no idea about the risk of blood transfusion, however, those ideas about the said risk were identified as AIDs, Hepatitis B, Syphilis and Malaria by $90.48 \%, 42.38 \%, 5.24 \%$ and $5.24 \%$ respondents respectively. (Table-3) 
Table-1: Distribution of the Baseline characteristics of respondents $n=820$

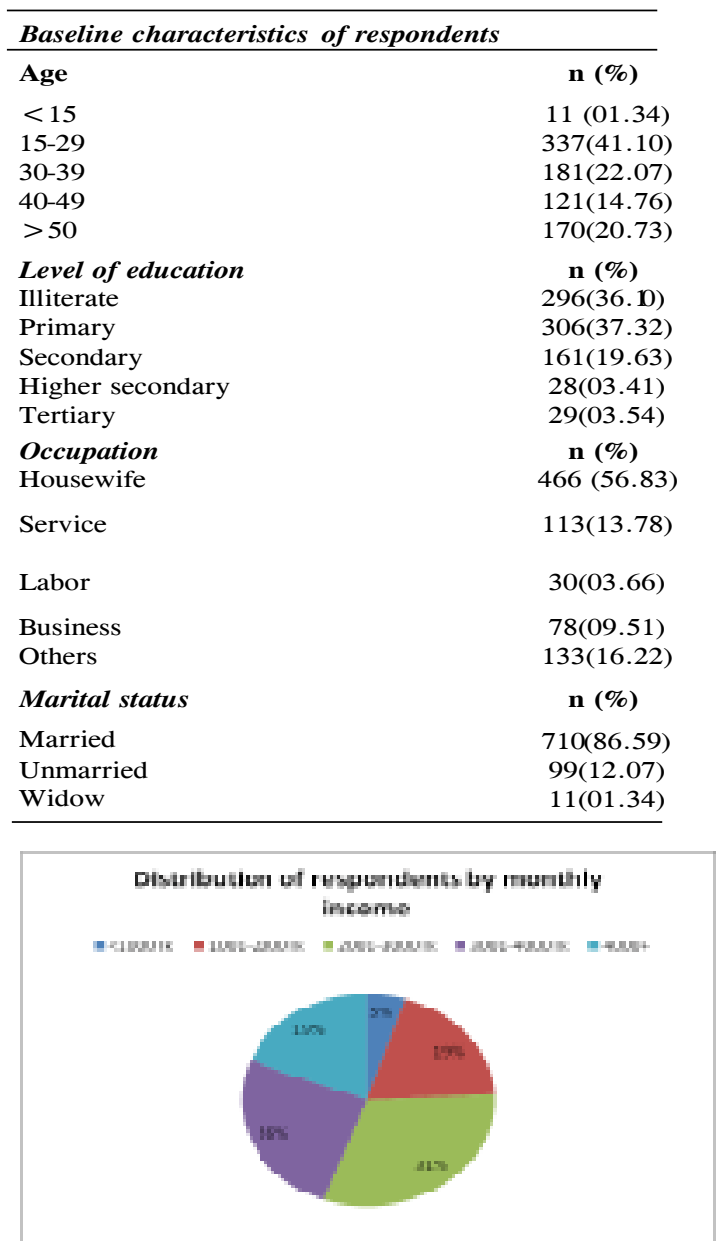

Figure-I: Pie diagram showing Distribution of espondents by monthly income

Table-II: Distribution of respondents by recipients perception related variables $\mathrm{n}=820$

\begin{tabular}{lcc}
\hline Recipients perception & Frequency (n) & Percentage (\%) \\
\hline $\begin{array}{l}\text { Idea about indication of blood } \\
\text { transfusion }\end{array}$ & \\
Yes & 495 & \\
No & 325 & 60.37 \\
Examinations usually performed & & 39.63 \\
before blood transfusion & 274 & \\
Blood grouping & 80 & 33.41 \\
Cross matching & 10 & 09.76 \\
Rh- typing & 456 & 01.22 \\
Not known & 171 & 55.61 \\
Idea about own blood group & 141 & \\
A & 72 & 20.85 \\
B & 219 & 17.20 \\
AB & 217 & 08.78 \\
O & & 26.71 \\
Not known & 142 & \\
Idea about persons who can donate & bood & \\
Male/female of any age & 244 & 17.31 \\
Male/female of specific age & 434 & 29.76 \\
Not known & & 52.93 \\
\hline
\end{tabular}

Figure-II: Bar diagram showing distribution of respondents by idea about frequency of blood donation in a year

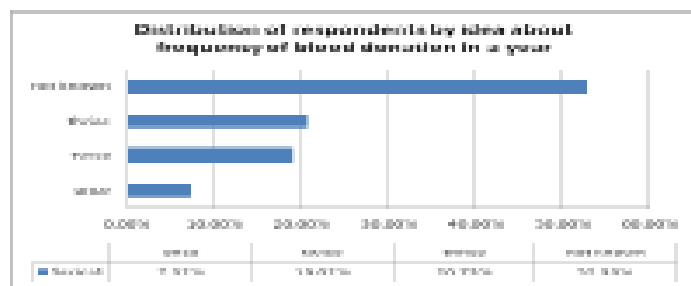

Table-III: Distribution of respondents by idea about blood donor selection and risk of blood transfusion $n=820$

\begin{tabular}{lcc}
\hline $\begin{array}{l}\text { Idea on donor selection and risk of } \\
\text { blood transfusion }\end{array}$ & & \\
& Frequency (n) & Percentage (\%) \\
\hline $\begin{array}{l}\text { Selection of blood donor for } \\
\text { transfusion }\end{array}$ & & \\
Professional donor & 128 & \\
Voluntary donor & 199 & 15.60 \\
Relatives & 302 & 24.27 \\
Others & 32 & 36.82 \\
Not knwn & 317 & 03.90 \\
Reasons for discouraging professional & & 38.66 \\
blood donors & & \\
Risk of blood transmissible diseases & 470 & \\
Poor quality blood & 69 & 57.32 \\
High cost & 00 & 08.41 \\
Not known & 281 & 00.00 \\
Idea about risk of blood transfusion & & 34.27 \\
Yes & 210 & \\
No & 610 & 25.61 \\
AIDs & 190 & 74.39 \\
Hepatitis B & 89 & 90.48 \\
Syphilis & 11 & 42.38 \\
Malaria & 11 & 05.24 \\
\hline & & 05.24 \\
\hline
\end{tabular}

\section{Discussion}

Blood transfusion can be life saving but in some instances it can cause dangerous harm to blood recipients. So it should not be given without definite indications. Blood is required in so many emergencies like accidents, acute surgery, acute medical emergency, burn and also in hematological conditions like thalassaemia, leukemia, aplastic anaemia etc. Both donor and recipients knowledge and awareness are vital in preventing transfusion transmissible diseases. In this study idea about indications of blood transfusion is found among $60.37 \%$ respondents.

However only $33.41 \%$ respondents identified usual examination as blood grouping performed before transfusion. There is scope for dissemination about the importance of various 
examinations before receiving blood among recipients. Moreover idea about own blood group is found among $73.54 \%$ respondents too which is encouraging.

The study also revealed $52.93 \%$ respondents were unaware about the persons who can donate blood. Moreover professional blood donors were the choice for own to only $15.60 \%$ respondents. Mass media and tertiary hospitals can play a vital role in creating awareness among the population and to the concern recipients about the persons who can donate blood and the risk associated with professional donors.

The respondents idea about transmissible diseases are identified as AIDs, Hepatitis B, Syphilis and Malaria by $90.48 \%, 42.38 \%$, $5.25 \%$ and $5.25 \%$ respectively. The findings will help gaining access to the recipients in order to motivate them for precautions need before receiving blood and choice of donors for his own and family members in future. Hepatitis $\mathrm{B}$ and $\mathrm{C}$ virus, Syphilis and other infectious agents such as Chagas disease infect many more recipients of blood products. ${ }^{3}$

Another study also revealed $29 \%$ professional donors carry Hepatitis B virus, 6\% carry Hepatitis C virus and $22 \%$ are positive for Syphilis. About $7 \%$ of general population are carrier for Hepatitis B virus. ${ }^{4}$ M S A Mansur ahmed et al conducted a study on Hepatitis B infection among Bangladeshi males seeking job abroad. In that study Hepatitis B infection was found among $6.5 \%$ of the study population. A statistically significant association was observed $(p<0.001)$ between exposure to parenteral injection and hepatitis B infection. The HBV infection was found to be 4.02 times greater for individuals exposed to parenteral injection then that of non-exposed. ${ }^{5}$

In a study, prevalence of transfusion transmissible diseases among blood donors attending the transfusion medicine department of Mitford Hospital Dhaka, Naher et al found that about $9 \%$ cases were transfusion transmissible diseases positive (HCV, HBV andSyphilis) and $4.0 \%, 2.1 \%$ and $3.0 \%$ respectively were positive for HbsAg, HCV and Syphilis. Needle sharing and tattoing were found among $4.0 \%$ and $0.9 \%$ donors. ${ }^{6}$ The safety measures at all levels particularly in selecting donors, blood screening as well as rational indication can be considered very carefully with due importance. This will add further benefit to the recipients in addition to his own knowledge and choice towards minimizing transfusion transmissible diseases in our situation. This will help in gaining confidence in receiving blood by the recipients and ensure safety in particular.

\section{Conclusion}

There is no substitute for blood and it is necessary in many emergencies both in acute medical and surgical conditions. In this study, recipients are found unaware about the persons who can donate blood. This is due to low level of education among the respondents. There is scope for dissemination about the importance of selection of donors among them. The study demands a large scale dissemination strategy as well as clear awareness program for the illiterate and moderately literate populations particularly the recipients receiving transfusion at tertiary care hospitals. It will help minimizing risk of transfusion transmissible disease both from donors and recipients side. An education and motivational program can be planned particularly for the younger and adult females and the program should address both recipients as well as donors with special emphasis on identification of high risk group, transfusion transmissible diseases and its prevention, importance of knowing own blood group, donor selection, common side effects as well as beneficial effects of blood donation.

\section{References}

1. Mackie M J, Ludlam C A and Haynes A P. Diseases of blood. In Haslett C, Chilvers ER, Hunter JAA and Boon NA. Davidson's Principles and Practice of Medicine 18th Edn Churchill Livingstone, Edinburg London New York Philadelphia Sydney Toronto: 1999 p 737-800.

2. Safe Blood Transfusion Program (SBTP)

3. WHO-AIDE-MEMORIE for National Blood Programs

4. 'Safe Blood Transfusion' prepared by blood transfusion subcommittee of National AIDS Committee (NAC) of Bangladesh, February 1997.

5. Ahmed M MSA, Khan MH, Choudhury MZ, et al. Hepatitis B infection among Bangladeshi males seeking job abroad. JOPSOM 2002; 21(2): 13-19.

6. Nahar D, Rahman M, Nahar A, et al. Prevalence of transfusion transmissible diseases among blood donors attending the transfusion medicine department of Mitford hospital in Dhaka. JOPSOM 2001; 20(2): 21-26. 